\title{
The role of spatial frequency in cued shifts of attention between global and local forms
}

\author{
MARVIN R. LAMB \\ Califormia State University, Hayward, Califormia, \\ and Veterans Administration Medical Center, Martinez, California \\ and \\ E. WILLIAM YUND \\ Veterans Administration Medical Center, Martinez, Califormia
}

\begin{abstract}
It has been suggested that shifts of attention between global and local forms might be based on selection between, or differential activation of, low- and high-spatial-frequency channels. In the present study, pretrial cues indicated which level (global or local) was likely to contain the target on each trial. There was a response time (RT) advantage for validly cued trials and an RT cost for invalidly cued trials relative to a neutral cue baseline. This cuing effect was the same for broadband stimuli and for contrastbalanced stimuli in which low spatial frequencies were eliminated. Thus, cued attentional shifts between global and local forms occur even when selection cannot be based on spatial frequency.
\end{abstract}

There is a great deal of evidence that the efficiency with which global and local level forms are analyzed can be modulated by attention. In fact, there is evidence that more than one such attentional mechanism exists (Lamb \& Robertson, 1988; Robertson, Egly, Lamb, \& Kerth, 1993). The two primary candidates offered by investigators have been a mechanism based on selection by spatial frequency or a mechanism based on selection by spatial location and/or extent. The latter hypothesis holds that the efficiency with which global and local forms are identified is affected by variations in the diameter of an attentional "spotlight" (Eriksen \& St. James, 1986; Lamb \& Robertson, 1988; Robertson et al., 1993; Stoffer, 1993). According to this hypothesis, the processing of global targets is facilitated when the attended area is relatively large (the size of a global form), whereas the processing of local targets is facilitated when the attended area is relatively small (the size of a local form). This has been referred to as regional selection (Robertson et al., 1993).

It has also been suggested that there might be another "categorical" attentional mechanism (Lamb \& Robertson, 1988; Robertson et al., 1993). According to this hypothesis, selection is based on some attribute that could serve to distinguish global forms from local forms. The most

This research was supported by the Medical Research Service of the Department of Veterans Affairs and by National Institute of Neurological Disorders and Stroke Grant NS27902 to M.R.L. The authors thank Russell L. De Valois for the use of his equipment to perform the Fourier transforms of the stimuli, Richard "Polo" Webster for computer programming, and Giti Zahir for her help with data collection. The authors also thank John Henderson, Lynn Robertson, and Lawrence Ward for helpful comments on an earlier version of this manuscript. Correspondence should be addressed to M. R. Lamb, Department of Psychology, California State University, Hayward, CA 94542 (e-mail: mlamb@ csuhayward.edu) studied candidate for such a categorical attribute is spatial frequency (Lamb \& Yund, 1993, 1996a; Lamb, Yund, \& Pond, 1999; Robertson, 1996; Shulman \& Wilson, 1987). The spatial frequency hypothesis holds that the processing of global targets is facilitated by the activation of low-spatial-frequency channels, whereas the processing of local targets is facilitated by the activation of highspatial-frequency channels. While there has been little dispute that "categorical" selection occurs, the hypothesis that spatial frequency is the basis of categorical selection is controversial (Lamb \& Yund, 1993, 1996a; Lamb et al., 1999; Robertson, 1996, 1999).

Three basic paradigms have been used to study shifts of attention between different levels of hierarchical structure. In the biasing procedure, shifts of attention are induced by manipulating the probability with which targets occur at each level. For example, in one condition, targets would be more likely to appear at the local level (local bias), and, in another condition, targets would be more likely to appear at the global level (global bias). Response times (RTs) are faster to global targets than to local targets in the global bias condition, whereas this pattern is reversed in the local bias condition. This performance tradeoff has been argued to reflect shifts of attention that occur in response to the different target level probabilities in the two conditions (Kinchla, Solis-Macias, \& Hoffman, 1983; Lamb \& Robertson, 1987; Lamb \& Yund, 1993; Robertson et al., 1993).

Lamb and Yund (1993) tested the spatial frequency hypothesis using the biasing procedure. Participants viewed broadband letter patterns typical of those used in the study of hierarchical stimulus processing, or they viewed contrast-balanced letter patterns that lacked low spatial frequencies. Because contrast-balanced stimuli lack low spatial frequencies, both local and global forms must be 
identified on the basis of high-spatial-frequency information. Thus, if the effect of biasing results from differential activation of high- versus low-spatial-frequency channels in the local and global bias conditions, then the biasing effect should be reduced or eliminated with contrast-balanced stimuli. Lamb and Yund (1993) found that the performance tradeoff was unaffected by the spatial frequency content of the stimulus. These data show that attentional selection between local and global forms, at least as measured with the biasing procedure, can occur even when selection by spatial frequency is not possible.

The second method that has been used to study shifts of attention between levels of hierarchical structure is the level repetition procedure. Many investigators have found that target identification is faster on trial $N$ if a target has appeared at the same level on trial $N-1$ (Filoteo et al., 1994; Filoteo et al., 1992; Hübner, 1997; Lamb, London, Pond, \& Whitt, 1998; Lamb, Pond, \& Zahir, 2000; Lamb \& Yund, 1996a; Lamb et al., 1999; Robertson, 1996; Ward, 1982, 1985). It has been argued that participants direct their attention to the target level on trial $n-1$, and identification of the target is facilitated if the target appears at the same (already attended) level on trial $n$.

Lamb and Yund (1996a) tested the spatial frequency hypothesis using the level repetition procedure. Again, participants viewed broadband or contrast-balanced letter patterns in separate blocks of trials, and, again, no evidence for attentional selection based on spatial frequency was obtained. The beneficial effect of level repetition was just as large for contrast-balanced stimuli as for broadband stimuli. In contrast, Robertson (1996) found no level repetition effect when broadband and contrast-balanced stimuli were alternated within a block of trials. However, Lamb et al. (1999) found a normal level repetition effect even under these circumstances as long as participants were experienced with contrast-balanced stimuli. Thus, attentional selection between local and global forms, at least as measured with the level repetition procedure, does not require differences in spatial frequency between local and global forms.

The third method that has been used to study shifts of attention between levels of hierarchical structure is the cuing procedure. In this case, a cue is presented prior to each trial indicating which level is likely to contain the target on that trial. It has been found that RTs are faster on validly cued trials and slower on invalidly cued trials relative to neutral baseline trials (Lamb et al., 2000; Stoffer, 1993, 1994).

In the present experiment, we examined whether attentional selection, as measured by the cuing procedure, requires differences in spatial frequency between global and local forms. There is evidence that cued shifts of attention invoke an attentional mechanism different from that invoked by level repetition and possibly by biasing. Recent findings suggest that the benefit derived from level repetition results from automatic (or stimulusdriven) shifts of attention that are outside voluntary control (Lamb et al., 1998; Lamb et al., 2000). The biasing procedure, on the other hand, is ambiguous with respect to the distinction between automatic and controlled (or goal-directed) shifts of attention. With the biasing procedure, participants may detect (or are sometimes explicitly instructed) that targets are more likely to occur at the biased level and thus may voluntarily direct attention to the biased level. However, the differing target probabilities for the two levels necessarily result in more level repetitions for the biased level than for the unbiased level. Thus, the biasing effect could instead be due to automatic shifts of attention driven by the different numbers of level repetitions at the biased and unbiased levels. Thus, with the biasing procedure, it is not possible to know whether the effects observed result from a controlled process, an automatic process, or some combination of the two. In contrast, at least some versions of the cuing procedure (i.e., those in which the cues symbolically represent the level cued) would seem to involve purely controlled shifts of attention (Lamb et al., 2000; Stoffer, 1993). Thus, the cuing procedure offers the possibility of clearly distinguishing the effects of controlled shifts of attention (due to the cues) and automatic shifts of attention (due to level repetition). Since different attentional mechanisms seem to be involved, spatial frequency might serve as the basis for attentional selection resulting from cues, even though this seems not to be the case for attentional selection resulting from level repetition (and biasing).

\section{EXPERIMENT 1}

The primary goal of the present experiments was to determine whether shifts of attention induced by pretrial cues are based on spatial frequency. That is, does a cue indicating the target level benefit performance via the differential activation of low-versus high-spatial-frequency channels? Each trial was preceded by a cue that accurately informed the participant as to which level would contain the target on that trial (valid cue), misinformed the participant as to which level would contain the target on that trial (invalid cue), or was noninformative as to target level (neutral cue). If the cues induce shifts of attention, then performance should be best with valid cues, intermediate with neutral cues, and poorest with invalid cues. In addition, half of the participants received broadband stimuli typical of those used in studies of hierarchical stimulus processing, whereas the other half received contrast-balanced stimuli such as those used to study the role of spatial frequency in hierarchical stimulus processing (Hughes, Fendrich, \& Reuter-Lorenz, 1990; Lamb \& Yund, 1993, 1996a, 1996b; Lamb et al., 1999; Robertson, 1996). Contrast-balanced stimuli lack low spatial frequencies so that both global and local forms must be identified on the basis of high-spatial-frequency information. Thus, if cuing derives its effect from the differential activation of high- versus low-spatial-frequency channels, then the cuing effect should be reduced or eliminated with contrast-balanced stimuli. 


\section{Method}

Participants. The participants received course credit at California State University, Hayward, for their participation. All participants gave informed consent. They also reported being right-handed and having normal or corrected-to-normal visual acuity. The $29 \mathrm{fe}-$ male and 23 male participants ranged in age from 18 to 34 years $(M=23.3, S D=4.8)$.

Apparatus. The visual stimuli were generated on a NEC XV17+ color monitor controlled by a Pentium-based microcomputer with an enhanced VGA graphics card. Timing of the visual stimuli was tied to the vertical sync pulse (refresh rate approximately $60 \mathrm{~Hz}$ ). All other events (responses, intertrial interval [ITI], etc.) were timed using the 8253 chip set to a 1 -msec time base. The status of the response keys (two push-button micro switches of a Gravis game controller) was monitored via the game port.

Stimuli. A small $\left(0.18^{\circ}\right)$ black $\left(12-\mathrm{cd} / \mathrm{m}^{2}\right)$ square presented in the center of the screen served as the fixation point. Hierarchically organized letter patterns like those in Figure 1 were presented above or below the fixation point $\left(2.0^{\circ}\right.$ from fixation to the nearest edge of the stimulus pattern). Global letters were constructed from the appropriate placement of local letters in a $5 \times 5$ matrix. Global letters were $3.2^{\circ}$ high and $2.0^{\circ}$ wide. Local letters were $0.44^{\circ}$ high and $0.27^{\circ}$ wide. The letters " $H$ " and " $S$ " served as targets, and the letters "A" and "E" served as distractors. Each hierarchical stimulus contained one target and one distractor letter, resulting in eight stimuli.

All stimuli were presented on a gray $\left(46-\mathrm{cd} / \mathrm{m}^{2}\right)$ background. Broadband stimuli (see Figure 1A) were composed of lines that were brighter $\left(66.5 \mathrm{~cd} / \mathrm{m}^{2}\right)$ than the background. Contrast-balanced stimuli (see Figure 1B) were identical to broadband stimuli except that the bright lines composing each local letter were surrounded by lines that were darker $\left(36.1 \mathrm{~cd} / \mathrm{m}^{2}\right)$ than the background. The luminance levels of the bright and dark lines were chosen so that the space-averaged luminance of the contrast-balanced stimuli would equal that of the background. Thus, the change in luminance from background was approximately twice as great for bright lines as for dark lines because dark lines occupied twice as much area as bright ones (see Figure 1B).
A two-dimensional Fourier transformation of representative broadband and contrast-balanced stimuli was performed (see Figure 2). Figure $2 \mathrm{~A}$ shows the transform for the broadband stimulus shown in Figure 1A. As can be seen, broadband stimuli contained a broad spectrum of spatial frequencies, with power concentrated near horizontal and vertical orientations. Figure $2 \mathrm{~B}$ shows the transform for the contrast-balanced stimulus shown in Figure 1B. This figure shows that the contrast-balanced stimuli had much less power at low spatial frequencies than did the broadband stimuli. More specifically, the contrast-balanced stimuli had virtually no power at spatial frequencies below 3 cycles/degree (i.e., the area within the inner circle in Figure 2) and very little power even out as far as 6 cycles/degree (i.e., the area between the two circles in Figure 2). Figure 2 represents a very conservative estimate of the effect of contrast balancing. All spatial frequencies for a given stimulus with contrast above .0006 are plotted. Since threshold for all spatial frequencies is above .001 at these luminance levels (see De Valois \& De Valois, 1990), all above-threshold spatial frequencies contained in the stimulus are plotted in Figure 2, and, in fact, some of the plotted points are well below threshold. The effect of contrast balancing illustrated in Figure 2 is representative of the effect of contrast balancing for all the stimuli used in this experiment.

A small rectangular box (approximately the size of one local letter) served as a local cue, a large rectangular box (approximately the size of one global letter) served as a global cue, and both rectangles presented simultaneously served as a neutral cue. Cues appeared centered around the fixation point. The lines composing the cues were brighter $\left(67 \mathrm{~cd} / \mathrm{m}^{2}\right)$ than the background.

The lines composing both the cues and the hierarchical stimuli were approximately $0.009^{\circ}$ thick. All luminance measurements were taken with a Minolta CS-100 Chroma Meter.

Procedure. Data were collected in two blocks of 160 trials each, with a short rest break between each block. The participants also received two practice blocks of 20 trials each prior to data collection.

The distance between the participant and the monitor screen was fixed at $65 \mathrm{~cm}$ by the use of a chinrest head restraint. Each trial began with a $500-\mathrm{msec}$ tone, followed immediately by the fixation

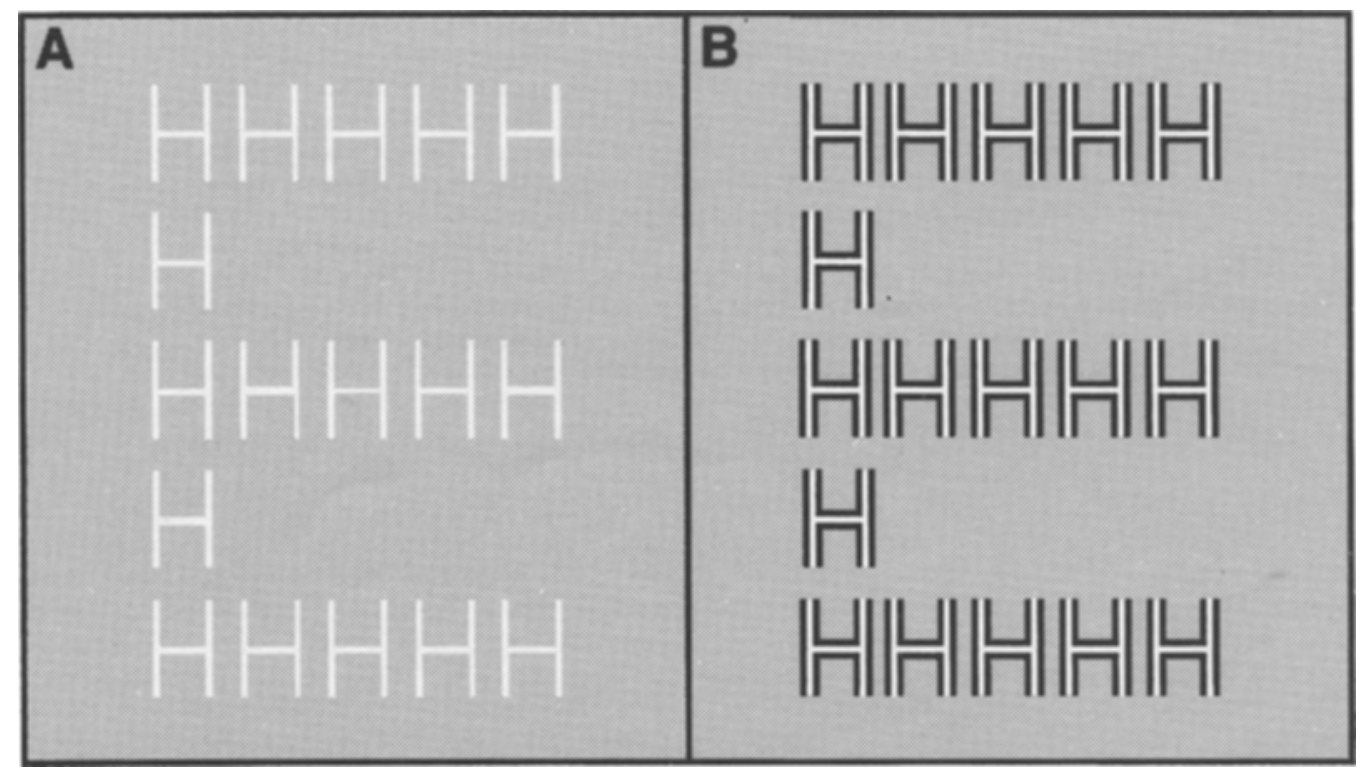

Figure 1. Drawings of a broadband stimulus (A) and a contrast-balanced stimulus (B). The precise spatial frequency content of the stimuli depends critically on luminance levels, which are not accurately reproduced in this figure. See text for details. 


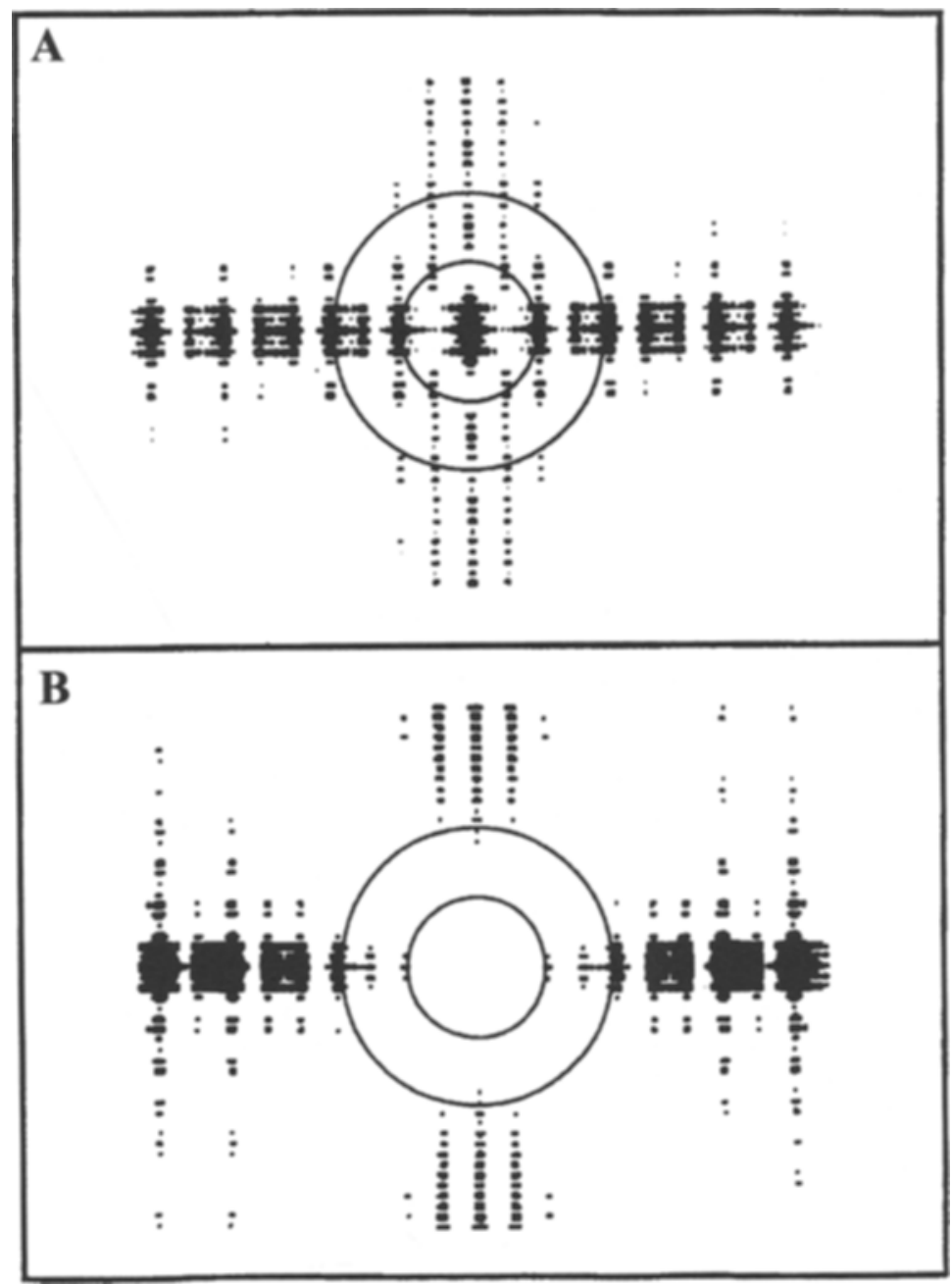

Figure 2. Two-dimensional Fourier transformations of the stimuli shown in Figure 1. (A) The transform for the broadband stimulus shown in Figure $1 \mathrm{~A}$. (B) The transform for the contrast-balanced stimulus shown in Figure 1B. The transforms are plotted in polar coordinates with spatial frequency on the radial dimension and orientation on the angular dimension. Spatial frequency increases linearly from zero at the center. The radii of the inner and outer circles are at 3 and 6 cycles/degree, respectively. The horizontal meridian of the polar coordinates indicates horizontal spatial frequency components, and the vertical meridian indicates vertical spatial frequency components. The size of each plotted point indicates the power of a given spatial frequency at a given orientation. When stimuli contain substantial power at many similar spatial frequencies and orientations plotted points overlap and produce larger filled areas.

point. The participants were instructed to look directly at the fixation point and not to move their eyes. A 500 -msec cue was presented $500 \mathrm{msec}$ after fixation onset. The fixation point remained on the screen for $500 \mathrm{msec}$, following cue offset and was then replaced by a 100 -msec presentation of one of the hierarchical letter patterns. The participants used the right hand to indicate which of the target letters ("H" or "S") was present on each trial. They pressed the "H" key with the index finger and the " $S$ " key with the middle finger. The participants were instructed to respond as quickly as possible while minimizing errors. There was a 1-sec ITI.

Global cues were followed by global targets $75 \%$ of the time (valid) and by local targets $25 \%$ of the time (invalid). Likewise local cues were followed by local targets $75 \%$ of the time (valid) and by global targets $25 \%$ of the time (invalid). Neutral cues were followed by global and local targets with equal probability. For each data block, $60 \%$ of the trials were validly cued, $20 \%$ were invalidly cued, and $20 \%$ were neutrally cued. A repeated-level trial was defined as any trial $(n)$ in which the preceding trial $(n-1)$ had contained a target at the same level. A changed-level trial was defined as any trial in which targets occurred at different levels on trials $n$ and $n-1$. Cue validity (valid, neutral, and invalid), level repetition (repeated level and changed level), target level (local and global), target letter ("H" and "S"), distractor letter ("A" and "E"), and stimulus location (above and below fixation) were completely counterbalanced within each block of data trials. Stimuli were presented randomly, with the restriction that no value from any of the above six factors could re- 
peat on more than four consecutive trials. Half of the participants received broadband letter patterns, and the other half received contrast-balanced letter patterns.

\section{Results and Discussion}

The data (both RTs and errors) were subjected to an analysis of variance (ANOVA) for mixed designs with target level (local and global), cue validity (valid, neutral, and invalid), and level repetition (repeated level and changed level) as repeated measures factors and frequency content (broadband and contrast balanced) as a betweensubjects factor. Mean percentage errors were calculated for each cell in the design for each participant. Median RTs (correct trials only) were calculated for each cell in the design for each participant, and the RT data reported are means of those medians.

Response time. There was a significant main effect of target level $\left[F(1,50)=28.41, M S_{\mathrm{e}}=22,084, p<.001\right]$, reflecting the fact that global targets were identified faster than local targets. The main effect of frequency content was not significant, but the frequency content $X$ level interaction was significant $\left[F(1,50)=5.57, M S_{\mathrm{e}}=\right.$ $22,084, p<.05]$. This was because contrast balancing slowed RTs when the participants were identifying global targets $\left[F(1,50)=4.21, M S_{\mathrm{e}}=88,538, p<.05\right]$, but not when they were identifying local targets $(F<1)$. Thus, consistent with numerous earlier studies, eliminating low spatial frequencies from the stimulus seems to have slowed global processing but had little, if any, effect on local processing (LaGasse, 1993; Lamb \& Yund, 1993, 1996a, 1996b; Lamb et al., 1999).

There was a main effect of cue validity $[F(2,100)=$ 20.67, $\left.M S_{\mathrm{e}}=16,913, p<.001\right]$ (see Figures 3A and 3B). RTs were faster following valid cues than following neutral cues $\left[F(1,50)=17.12, M S_{\mathrm{e}}=5,633, p<.001\right]$, faster following neutral cues than following invalid cues $\left[F(1,50)=15.23, M S_{\mathrm{e}}=17,562, p<.001\right]$, and faster following valid cues than following invalid cues $[F(1,50)=$ $\left.24.87, M S_{\mathrm{e}}=27,543, p<.001\right]$. In other words, valid cues produced benefits and invalid cues produced costs relative to the neutral cue baseline. Thus, the cues seem to have been effective in initiating shifts of attention to the cued level.

There was no indication that the spatial frequency content of the stimuli reduced the ability of the cues to produce shifts of attention. The frequency content $\times$ cue validity interaction was nonsignificant $[F(2,100)=1.58$, $\left.M S_{\mathrm{e}}=16,913\right]$. In addition, the effect of cue validity was

\section{Broadband Contrast Balanced}

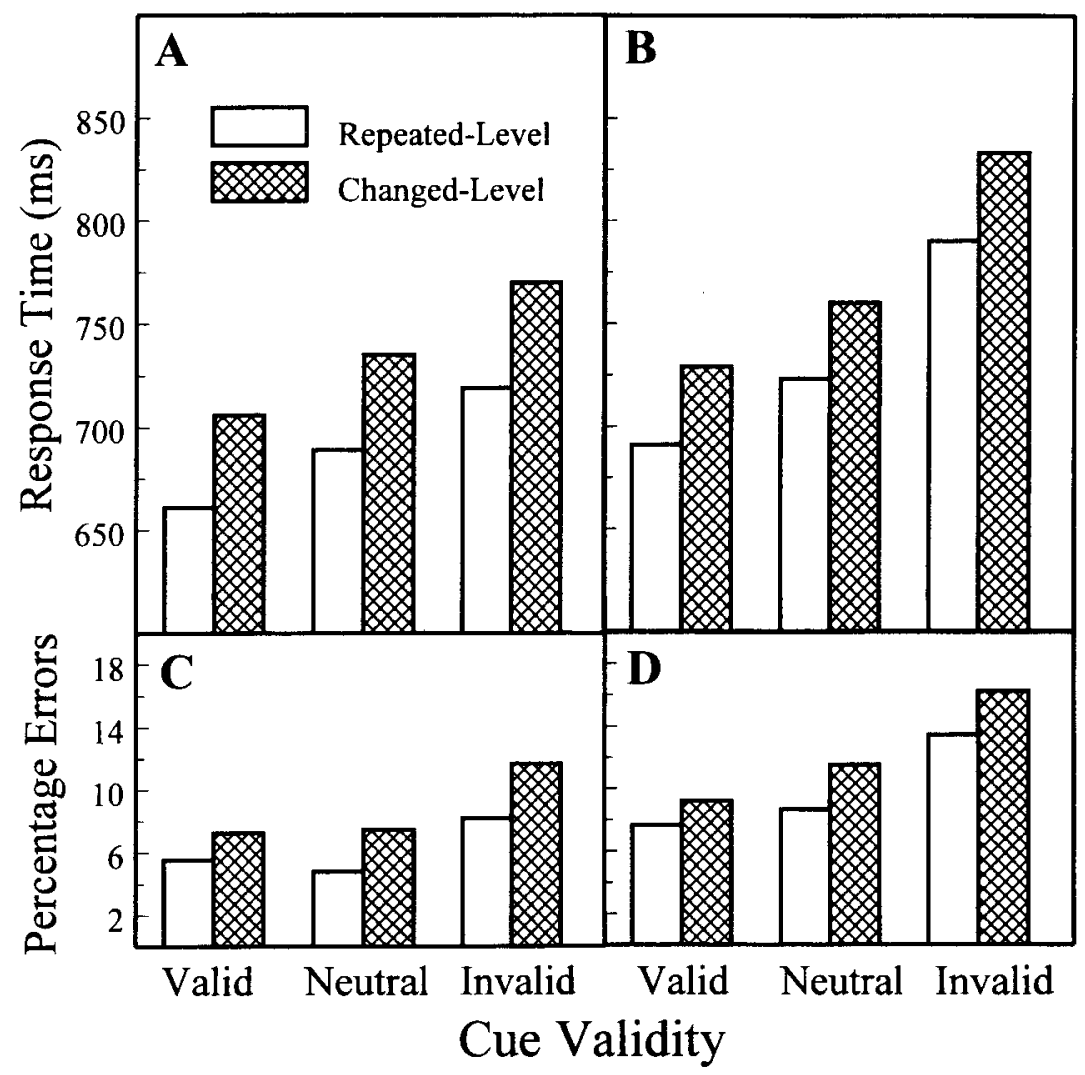

Figure 3. Response time ( $A$ and $B$ ) and percentage errors $(C$ and $D$ ) for repeated-level (open bars) and changed-level (filled bars) trials as a function of cue validity. Data are shown separately for broadband ( $A$ and $C$ ) and contrast-balanced ( $B$ and $D$ ) stimuli. 
significant for contrast-balanced stimuli $[F(2,50)=12.58$, $\left.M S_{\mathrm{e}}=22,266, p<.001\right]$ and for broadband stimuli $\left[F(2,50)=8.33, M S_{\mathrm{e}}=11,560, p<.001\right]$. Thus, the present data provide no support for the hypothesis that the shifts of attention initiated by the cues was based on differential activation of high- versus low-spatial-frequency channels.

The main effect of level repetition was also significant $\left[F(1,50)=87.58, M S_{\mathrm{e}}=3,330, p<.001\right]$, reflecting the fact that RTs were faster on repeated-level trials than on changed-level trials. Consistent with earlier studies (Lamb \& Yund, 1996a; Lamb et al., 1999), this level repetition effect was also unaffected by contrast balancing. The frequency content $\times$ level interaction was nonsignificant $(F<1)$, and the beneficial effect of level repetition was present for contrast-balanced stimuli $\left[F(1,25)=26.44, M S_{\mathrm{e}}=4,522, p<.001\right]$ and for broadband stimuli $\left[F(1,25)=81.69, M S_{\mathrm{e}}=2,138, p<.001\right]$. Thus, again, the present data provide no support for the hypothesis that shifts of attention, as measured by the level repetition effect, are based on spatial frequency.

Since contrast balancing affects mostly global RT, we examined the data to see whether eliminating low spatial frequencies affected the cue validity and level repetition effects specifically when the participants were identifying targets at the global level. Again, the effects of cue validity $\left[F(2,100)=14.07, M S_{\mathrm{e}}=11,329, p<.001\right]$ and level repetition $\left[F(1,50)=48.84, M S_{\mathrm{e}}=2,758, p<\right.$ $.001]$ were significant, and neither of these factors interacted with frequency content.

There was no hint of a cue validity $\times$ level repetition interaction $(F<1)$. Thus, cuing and level repetition seem to have exerted independent effects on the processing of global and local forms. These data are consistent with earlier evidence suggesting that the beneficial effect of level repetition derives from an automatic process that is outside the participant's voluntary control (Lamb et al., 1998; Lamb et al., 2000; Ward, 1985). If the poor performance on neutral-cue changed-level trials were the result of the inability to predict which level would contain the target and thus know in advance where to direct attention, then the difference between repeated-level and changed-level trials should disappear on valid- and invalid-cue trials. On valid-cue trials, participants should always be attending to the appropriate level regardless of level repetition; on invalid-cue trials, participants should never be attending to the appropriate level regardless of level repetition. In either case, there should be no difference in performance between repeated-level and changedlevel trials. Thus, the lack of an interaction between cue validity and level repetition suggests that there are two different mechanisms that affect the efficiency with which forms at different levels are processed - perhaps a controlled mechanism associated with cuing and an automatic process associated with level repetition. Furthermore, the fact that neither mechanism was affected by the elimination of low spatial frequencies suggests that neither is based on selection between, or differential activation of, high- and low-spatial-frequency channels.

Errors. The error data were consistent with the RT data (see Figures $3 \mathrm{C}$ and $3 \mathrm{D}$ ). There was a significant main effect of target level $\left[F(1,50)=21.06, M S_{\mathrm{e}}=1.7, p<\right.$ $.001]$, reflecting the fact that fewer errors were made to global targets than to local targets. The main effect of frequency content was significant $\left[F(1,50)=5.50, M S_{\mathrm{e}}=\right.$ $3.8, p<.05]$, reflecting the fact that fewer errors were made to broadband stimuli than to contrast-balanced stimuli. There was a main effect of cue validity $[F(1,50)=$ $\left.30.75, M S_{\mathrm{e}}=0.3, p<.001\right]$, a main effect of level repetition $\left[F(2,100)=16.63, M S_{\mathrm{e}}=0.9, p<.001\right]$, and no cue validity $\times$ level repetition interaction $(F<1)$. Finally, frequency content did not interact with any other factor.

\section{EXPERIMENT 2}

The fact that both cuing and level repetition influenced performance and, furthermore, that these factors did not interact suggests that two independent processes affect the efficiency with which global and local forms are processed. There is evidence that the beneficial effect of level repetition results from an automatic process that is outside voluntary control (Lamb et al., 1998; Lamb et al., 2000; Ward, 1985). It has also been suggested that, at least under some conditions, cues initiate controlled shifts of attention (Lamb et al., 2000; Stoffer, 1993). If so, the results of Experiment 1 show that neither automatic nor controlled shifts of attention rely on selection between spatial frequency channels. However, it could be argued that the cues in Experiment 1 initiated automatic, rather than controlled, shifts of attention. This is because the relation between the cues and the level cued was not arbitrary. Global cues were the same size as global targets, and local cues were the same size as local targets. As a result, it could be that processing the cue resulted in an automatic adjustment of the attended area to a size that was advantageous for the cued level (i.e., a large "spotlight" for valid-global trials and small "spotlight" for valid-local trials).

The main purpose of Experiment 2 was to determine whether the cues derived their effect from controlled or automatic attentional shifts. This was accomplished by reversing the meaning of the cues from that in Experiment 1. That is, large rectangles predicted local (rather than global) targets, and small rectangles predicted global (rather than local) targets. If the cues achieve their effect via an automatic adjustment in the size of the attended area, then performance should be determined by the size of the cue rather than by its symbolic meaning (i.e., which level it predicts). In contrast, if the cues achieve their effect via a controlled shift of attention, then performance should be determined by the symbolic meaning of the cue rather than by its size. 


\section{Method}

Participants. The participants received course credit at California State University, Hayward, for their participation. All participants gave informed consent. They also reported being right-handed and having normal or corrected-to-normal visual acuity. The $18 \mathrm{fe}$ male and 8 male participants ranged in age from 18 to 39 years $(M=22.0, S D=5.2)$.

Apparatus, Stimuli, and Procedure. The apparatus, stimuli, and procedure were the same as in Experiment 1 except that (1) the small rectangle served as the global cue and the large rectangle served as the local cue and (2) data were collected in a single block of 160 trials in which all hierarchical stimuli were contrast balanced.

\section{Results and Discussion}

The data (both RTs and errors) were subjected to an ANOVA, with target level (local and global), cue validity (valid, neutral, and invalid), and level repetition (repeated level and changed level) as repeated measures factors.

Response time. There was no indication that reversing the meaning of the cues altered their effect. There was a main effect of cue validity $\left[F(2,50)=14.59, M S_{\mathrm{e}}=\right.$ $17,148, p<.001$ ] (see Figure 4A). As in Experiment 1, RTs were faster following valid cues than following neutral cues $\left[F(1,25)=11.66, M S_{\mathrm{e}}=5,101, p<.01\right]$, faster following neutral cues than following invalid cues $\left[F(1,25)=11.88, M S_{\mathrm{e}}=17,272, p<.01\right]$, and faster fol- lowing valid cues than following invalid cues $[F(1,25)=$ $\left.16.71, M S_{\mathrm{e}}=29,072, p<.001\right]$. Combined with the findings from Experiment 1, this clearly shows that it was the symbolic meaning of the cues and not their size per se that produced the cuing effect. Thus, the cues in these experiments seem to have initiated controlled shifts of attention. Moreover, the cues were effective even though the stimuli were contrast balanced and thus lacking in low spatial frequencies. Thus, again, there was no indication that cued shifts of attention rely on selection by spatial frequency.

The main effect of level repetition was also significant $\left[F(1,25)=21.68, M S_{\mathrm{e}}=3,984, p<.001\right]$, reflecting the fact that RTs were faster on repeated-level trials than on changed-level trials, again despite the fact that low spatial frequencies were absent for these stimuli. Thus, there was no indication that shifts of attention, as measured by the level repetition effect, rely on spatial frequency.

As in Experiment 1, the cue validity $\times$ level repetition interaction was nonsignificant $\left[F(2,50)=1.69, M S_{\mathrm{e}}=\right.$ $4,640]$. However, visual inspection of the data suggests that the level repetition effect was reduced when cues were invalid (see Figure 4A). Indeed, whereas repeatedlevel trials were faster than changed-level trials for both valid-cue trials $\left[F(1,25)=37.82, M S_{\mathrm{e}}=1,481, p<.001\right]$ and neutral-cue trials $\left[F(1,25)=12.28, M S_{\mathrm{e}}=3,358\right.$,

\section{Contrast Balanced}

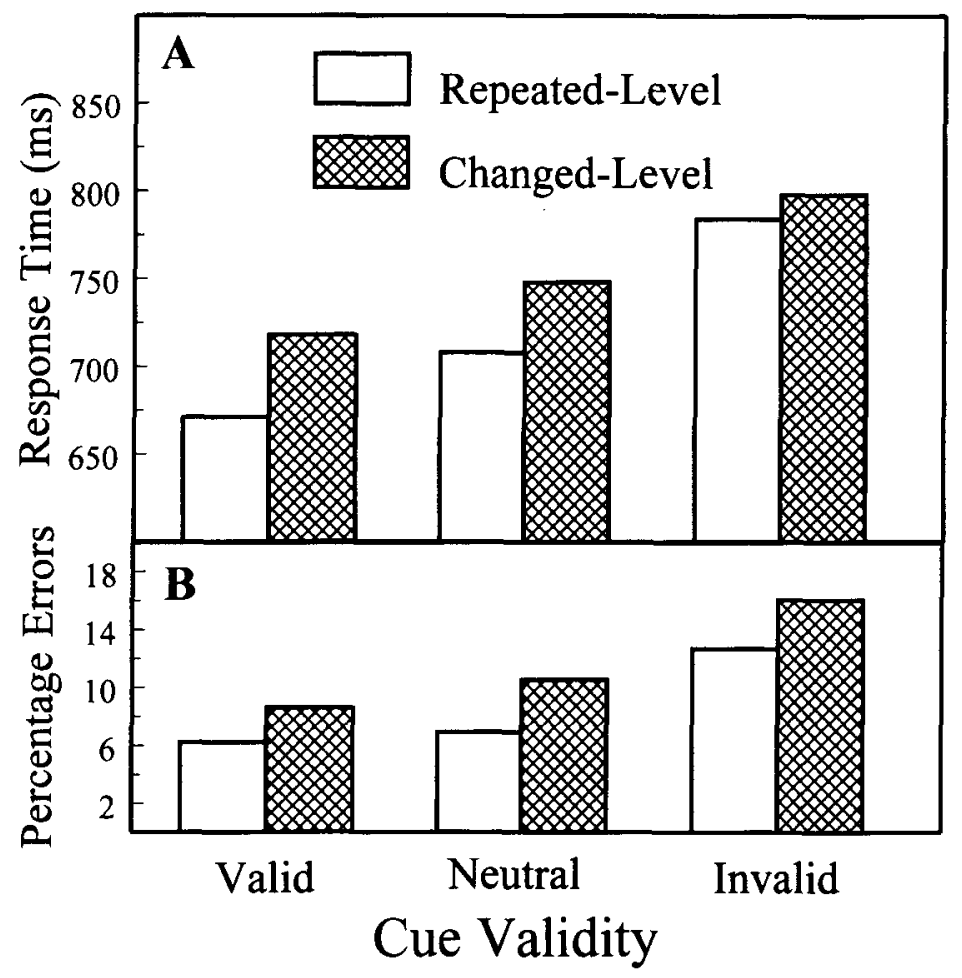

Figure 4. Response time (A) and percentage errors (B) for repeated-level (open bars) and changed-level (filled bars) trials as a function of cue validity. 
$p<.01$ ], this difference was nonsignificant for invalidcue trials. On the other hand, as discussed below, this difference was significant in the error data.

Errors. The error data for Experiment 2 are shown in Figure 4B. There was a main effect of target level $\left[F(1,25)=14.60, M S_{\mathrm{e}}=2.0, p<.001\right]$, reflecting the fact that fewer errors were made to global targets than to local targets. There was a main effect of cue validity $\left[F(2,50)=20.27, M S_{\mathrm{e}}=0.7, p<.001\right]$. There was also main effect of level repetition $\left[F(1,25)=11.73, M S_{\mathrm{e}}=\right.$ $0.7, p<.01]$. There was no indication of a cue validity $\times$ level repetition interaction $(F<1)$. In fact, the effect of level repetition was significant for valid-cue trials $\left[F(1,25)=13.44, M S_{\mathrm{e}}=0.1, p<.001\right]$, neutral-cue trials $\left[F(1,25)=7.94, M S_{\mathrm{e}}=0.4, p<.01\right]$, and invalid-cue trials $\left[F(1,25)=4.20, M S_{\mathrm{e}}=0.7, p<.05\right]$.

\section{GENERAL DISCUSSION}

The present data indicate that the cuing and level repetition effects result from the activity of two independent processes, one controlled and the other automatic. The fact that the cues could be flexibly interpreted (i.e., the meaning of the cues could be reversed) suggests that they initiated controlled rather than automatic shifts of attention. Moreover, the level repetition effect was unaltered by the controlled shifts of attention initiated by the cues. This is consistent with earlier data (Lamb et al., 1998; Lamb et al., 2000; Ward, 1985) suggesting that the level repetition effect is due to an automatic process that cannot be voluntarily overridden.

The present data show that shifts of attention between levels of hierarchical structure can occur even when selection based on spatial frequency is not possible. There was no suggestion in the data that eliminating low spatial frequencies altered the cuing effect or the level repetition effect. Thus, these data provide no support for the hypothesis that shifts of attention between levels of hierarchical structure are based on selection between high- and low-spatial-frequency channels, and, furthermore, this is the case whether these shifts are controlled or automatic.

It has been argued (Robertson, 1999) that attentional selection is based on spatial frequency for broadband stimuli but that other unknown attributes are substituted as the basis for selection whenever spatial frequency differences between global and local forms are not present (e.g., for contrast-balanced stimuli). This possibility cannot be ruled out with certainty, but this hypothesis does pose some difficulties. First, there has been no clear suggestion as to what these other attributes might be. However, it would seem reasonable to expect that some attributes would support selection more readily than others, so that a change in the attributes on which selection is based would be accompanied by some change in performance. However, there is no evidence that attentional selection, assessed in a wide variety of ways (i.e., biasing, level repetition, and cuing), is at all different when spatial frequency differences between global and local forms are present versus when they are absent. Moreover, the present data show that this is the case even when two different types of selection (i.e., controlled and automatic) are occurring simultaneously. Thus, although it is possible that selection is based on different, but equally effective, attributes when spatial frequency differences are present versus when they are absent, there is, in fact, no direct evidence that spatial frequency is ever the basis for selection between levels. On the other hand, there is a great deal of evidence that selection is just as effective when spatial frequency cannot serve as the basis for selection as when it, in principle, could.

Perhaps the most direct evidence supporting the spatial frequency hypothesis comes from a study by Shulman and Wilson (1987). These investigators induced participants to attend selectively to different levels of structure by having them identify local or global forms in separate blocks of trials. On a small number of probe trials, participants were also asked to detect sinusoidal gratings of different spatial frequencies. Low-frequency gratings were more easily detected than high-frequency gratings in global blocks, whereas the reverse was true in local blocks. While these data are consistent with the notion that attention to level is based on spatial frequency, as the authors point out, it could also be that participants adopted a small attentional spotlight in local blocks and a large attentional spotlight in global blocks. This could in turn have affected the efficiency with which the grating stimuli were processed. For example, a small attended area might be particularly disruptive for relatively lower spatial frequency gratings because fewer cycles would fit within the attended area. Thus, these data do not provide compelling evidence that selection is based on spatial frequency.

The spotlight hypothesis might also provide an account of the present data. For example, the attended area might increase in size in response to global cues and decrease in size in response to local cues. One potential difficulty with this hypothesis, however, is that, in the present experiments, the location of the hierarchical stimulus on each trial (above or below fixation) was randomly determined. Thus, it could be argued that adjusting the size of the spotlight in response to the cue would be of little value since there would be no way for the participant to anticipate where the spotlight should be directed until after the stimulus appeared (Lamb \& Yund, 1996a). However, this argument rests on the assumption that, because the target location is not known in advance, the spotlight never reaches that location. It could be, however, that the abrupt onset of the hierarchical stimulus produces a relatively rapid (stimulus-driven) movement of attention to the stimulus location, and, if the size of the attended area is already appropriate (e.g., due to valid cuing or level repetition), performance benefits. In contrast, if the size of the attended area is not appropriate at the time the stimulus appears (e.g., due to invalid cuing or level change), performance suffers (either because of a mismatch between the size of the attended area and the size of the target stimulus or because of the additional time required to 
adjust the size of the attended area to conform to the size of the target stimulus).

Lamb and Yund (1996a) offered another possible explanation of variations in processing efficiency for global and local forms that they called the mechanism activation hypothesis. This hypothesis is based in part on evidence that somewhat different neural mechanisms are associated with the processing of global-level and locallevel objects (Fink et al., 1996, 1997; Heinz \& Munte, 1993; Lamb, Robertson, \& Knight, 1990). The mechanism activation hypothesis holds that the neural mechanism associated with a given level is automatically activated when a target at that level is identified. By this account, level repetition benefits performance because identification of a target on the previous trial activates the appropriate neural mechanism for identification of a target at that same level on the current trial. If so, the present data suggest that the computations performed by these different neural mechanisms are not based on spatial frequency. If they were, the level repetition effect should have been eliminated by contrast balancing.

In conclusion, there is now a great deal of evidence showing that attentional selection between global and local forms occurs under conditions in which selection based on spatial frequency is not possible. This is true regardless of whether those shifts of attention are measured with the biasing procedure (Lamb \& Yund, 1993), the level-repetition procedure (Lamb \& Yund, 1996a; Lamb et al., 1999), or the cuing procedure (the present data). The present data show further that two of these procedures (level repetition and cuing) reflect the operation of different mechanisms that exert independent effects on the efficiency with which forms at different levels are analyzed. Neither of these mechanisms, however, require selection between, or differential activation of, high- and low-spatial-frequency channels.

\section{REFERENCES}

De VAlois, R. L., \& De ValoIs, K. K. (1990). Spatial vision. New York: Oxford University Press.

ERIKSEN, C. W., \& ST. JAMES, J. D. (1986). Visual attention within and around the field of focal attention: A zoom lens model. Perception \& Psychophysics, 40, 225-240.

Filoteo, J. V., Delis, D. C., Demadura, T. L., Salmon, D. P., Roman, M. J., \& ShULTS, C. W. (1994). Abnormally rapid disengagement of covert attention to global and local stimulus levels may underlie the visuospatial impairment in Parkinson's patients. Neuropsychology, 8 , 210-217.

Filoteo, J. V., Delis, D. C., Massman, P. J., Demadura, T. L., ButTERS, N., \& SAlMON, D. P. (1992). Directed and divided attention in Alzheimer's disease: Impairment in shifting attention to global and local stimuli. Journal of Clinical \& Experimental Neuropsychology, 14, 871-883.

Fink, G. R., Halligan, P. W., Marshall, J. C., Frith, C. D., FrackOWIAK, R. S. J., \& Dolan, R. J. (1996). Where in the brain does visual attention select the forest and the trees? Nature, 382, 626-628.

Fink, G. R., Halligan, P. W., Marshall, J. C., Frith, C. D., FrackOWIAK, R. S. J., \& DoLAN, R. J. (1997). Neural mechanisms involved in the processing of global and local aspects of hierarchically organized visual stimuli. Brain, 120, 1779-1791

HEINZ, H. J., \& MuNTE, T. F. (1993). Electrophysiological correlates of hierarchical stimulus processing: Dissociation between onset and later stages of global and local target processing. Neuropsychologia, 31, 841-852.

HüBNER, R. (1997). The effect of spatial frequency on global precedence and hemispheric differences. Perception \& Psychophysics, 59, 187-201.

Hughes, H. C., Fendrich, R., \& Reuter-Lorenz, P. A. (1990). Global versus local processing in the absence of low spatial frequencies. Journal of Cognitive Neuroscience, 2, 272-282.

Kinchla, R. A., Solis-Macias, V., \& Hoffman, J. (1983). Attending to different levels of structure in a visual image. Perception \& Psychophysics, 33, 1-10.

LaGasse, L. L. (1993). Effects of good form and spatial frequency on global precedence. Perception \& Psychophysics, 53, 89-105.

Lamb, M. R., London, B., Pond, H. M., \& Whitt, K. A. (1998). Automatic and controlled processes in the analysis of hierarchical structure. Psychological Science, 9, 14-19.

LAMb, M. R., Pond, H. M., \& ZAHIR, G. (2000). Contributions of automatic and controlled processes to the analysis of hierarchical structure. Journal of Experimental Psychology: Human Perception \& Performance, 26, 234-245.

LAMB, M. R., \& RoBERTSON, L. C. (1987). Effects of acute alcohol on attention and the processing of hierarchical patterns. Alcoholism: Clinical \& Experimental Research, 11, 243-248.

LAMB, M. R., \& RoBer TSON, L. C. (1988). The processing of hierarchical stimuli: Effects of retinal locus, locational uncertainty, and stimulus identity. Perception \& Psychophysics, 44, 172-181.

Lamb, M. R., Robertson, L. C., \& KNight, R. T. (1990). Component mechanisms underlying the processing of hierarchically organized patterns: Inferences from patients with unilateral cortical lesions. Journal of Experimental Psychology: Learning, Memory, \& Cognition, 16, 471-483.

LAMB, M. R., \& Yund, E. W. (1993). The role of spatial frequency in the processing of hierarchically organized structure. Perception $\&$ Psychophysics, 54, 773-784.

LAMB, M. R., \& YUND, E. W. (1996a). Spatial frequency and attention: Effects of level-, target-, and location-repetition on the processing of global and local forms. Perception \& Psychophysics, 58, 363-373.

LAMB, M. R., \& YUND, E. W. (1996b). Spatial frequency and interference between global and local levels of structure. Visual Cognition, 3, 193-219.

LAMB, M. R., Yund, E. W., \& Pond, H. M. (1999). Is attentional selection to different levels of hierarchical structure based on spatial frequency? Journal of Experimental Psychology: General, 128, 88-94.

RoBERTSON, L. C. (1996). Attentional persistence for features of hierarchical patterns. Journal of Experimental Psychology: General, 125, 227-249.

RoBERTSON, L. C. (1999). Spatial frequencies as a medium for guiding attention: Comment on Lamb, Yund, and Pond (1999). Journal of Experimental Psychology: General, 128, 95-98.

Robertson, L. C., Egly, R., LAmb, M. R., \& Kerth, L. (1993). Spatial attention and cuing to global and local levels of hierarchical structure. Journal of Experimental Psychology: Human Perception \& Performance, 19, 471-487.

Shulman, G. L., \& Wilson, J. (1987). Spatial frequency and selective attention to local and global information. Perception, 16, 89-101.

STOFFER, T. H. (1993). The time course of attentional zooming: A comparison of voluntary and involuntary allocation of attention to the levels of compound stimuli. Psychological Research, 56, 14-25.

STOFFER, T. H. (1994). Attentional zooming and the global-dominance phenomenon: Effects of level-specific cueing and abrupt visual onset. Psychological Research, 56, 83-98.

WARD, L. M. (1982). Determinants of attention to local and global features of visual forms. Journal of Experimental Psychology: Human Perception \& Performance, 8, 562-581.

WARD, L. M. (1985). Covert focussing of the attentional gaze. Canadian Journal of Psychology, 39, 546-563.

(Manuscript received July 2, 1998; revision accepted for publication May 5,1999 .) 